\title{
„Früher waren wir die Exoten“ - Potenziale von Migranten aus islamisch geprägten Herkunftsländern
}

\author{
Tayfun Keltek und Engin Sakal
}

\section{Vom Gastarbeiter zum „Mensch mit Migrationshintergrund“ ${ }^{1}$}

Dieser Beitrag soll einen allgemeinen Überblick über die Bevölkerung mit Migrationshintergrund $(\mathrm{MH})$ insgesamt geben. Dabei sollen Angaben sowohl zu Migranten, Eingebürgerten und zu ausländischen Staatsangehörigen in Deutschland gemacht werden. Sie sollen dazu dienen, sich einen Gesamteindruck zu verschaffen, und statistische Angaben ausgewählter Bereiche runden das Bild ab. Die Angaben erheben nicht den Anspruch auf Vollständigkeit für alle Lebensbereiche. Es sollen lediglich zentrale Faktoren dargestellt und grundlegende Informationen geliefert werden.

Bedingt durch die Größe der Gruppe der Migranten werden nähere Angaben zu der Gruppe mit türkischem Migrationshintergrund für Vergleiche vorgenommen. Zudem sind das Gros der Türkischstämmigen Muslime, was jeweils einen besonderen Blick auch auf das in diesem Band fokussierte Religionsmerkmal erlaubt.

Die Migranten, auch die mit türkischem Hintergrund, sind als Unternehmer, Arbeitnehmer, Schüler, Studenten und letztendlich als Konsumenten mit ihrem Anteil an der Gesellschaft zum bedeutenden Faktor geworden. Den Abschluss des Beitrages bilden Biographien von drei in Deutschland lebenden unterschiedlichen Migranten, zu denen Interviews geführt wurden.

\footnotetext{
${ }^{1}$ Der Begriff Mensch mit „Migrationshintergrund“ hat in diesem Beitrag keinerlei diskriminierende Aussagekraft. Er ist in der statistischen Erfassung von Daten geläufig, auch das Statistische Bundesamt benutzt diesen Begriff für die Datenerhebung. Und entsprechend findet er in diesem Beitrag Verwendung.
} 
Die jüngere Zuwanderungsgeschichte nach Deutschland beginnt nach der Gründung der Bundesrepublik Deutschland. Das Wirtschaftswachstum nach dem Zweiten Weltkrieg führte zu einem hohem Arbeitskräftebedarf in Deutschland. Bedingt durch den Mauerbau wurde der Arbeitskräftestrom aus dem Osten unterbrochen. Deshalb schloss die Bundesregierung zwischen 1955 und 1973 sogenannte Anwerbeabkommen für ausländische Arbeitskräfte mit ausländischen Regierungen ab, unter ihnen auch mit Regierungen islamisch geprägter Staaten. $\mathrm{Zu}$ den Anwerbeländern gehörten neben Griechenland, Italien, Portugal, Spanien, Südkorea auch das damalige Jugoslawien, Marokko, Tunesien und die Türkei.

Die anfängliche Vorstellung der Bundesrepublik Deutschland bei der Anwerbung von Gastarbeitern war, dass sich die Menschen zeitlich beschränkt in Deutschland aufhalten und später wieder in ihre Heimatländer zurückkehren und andere, neue Arbeitskräfte, im Austausch nach Deutschland kommen würden. Diese Absicht konnte jedoch nicht verwirklicht werden, da viele Unternehmen kein Interesse an größeren Fluktuationen unter ihrer Belegschaft hatten. Zudem wären die Kosten dafür zu hoch und damit unwirtschaftlich gewesen. Die Bundesregierung entsprach deshalb dem Wunsch der Unternehmen, die angelernten Kräfte längere Zeit in Deutschland zu belassen, und die „Gastarbeiter“ blieben damit länger als vorgesehen in der Bundesrepublik Deutschland.

Erst die Rezession und die Ölkrise Anfang der 70er Jahre führten 1973 zum Anwerbestopp ausländischer Arbeitskräfte. Eine Ausnahme bildete das Abkommen zur Anwerbung von Pflegepersonal aus Südkorea. Krankenschwestern aus Südkorea wurden auch nach dem Anwerbestopp von 1973 weiter angeworben. Sie sollten zunächst für drei Jahre nach Deutschland kommen, aber auch sie blieben länger. So leben heute viele Arbeitsmigranten und deren Nachkommen dauerhaft in Deutschland. ${ }^{2}$

Ende 1980 war die ausländische Bevölkerung in Deutschland auf 4,5 Millionen angestiegen. ${ }^{3}$ Aussagen wie „Mir kommt kein Türke mehr über die Grenze“, zu der sich der damalige Bundeskanzler Helmut Schmidt in einem Interview mit

\footnotetext{
${ }^{2}$ In diesem Beitrag wird einheitlich eine nicht geschlechterspezifizierende Bezeichnung verwendet. Mit Arbeiter, Schüler, Migrant und Kollege sind immer Männer und Frauen gemeint.

${ }^{3}$ Vgl. Statistisches Bundesamt, 2012/1.
} 
der Zeitung Die Zeit am 5. Februar 1982 verstieg, war für die Situation bezeichnend. In der Koalitionsvereinbarung von 1982 zwischen der CDU/CSU und FDP heißt es unter dem Kapitel Ausländerpolitik: „Die Bundesrepublik ist kein Einwanderungsland." Derartige Aussagen gaben das politische Klima in Deutschland lange Zeit unmissverständlich wider. Doch die gesellschaftlichen Realitäten waren anders. Die Anzahl der in Deutschland lebenden Ausländer stieg in den darauffolgenden Jahren im Jahr 1990 auf 5,5 Millionen, und bis 2000 waren es bereits 7,2 Millionen. ${ }^{4}$ Es dauerte relativ lange, bis sich auch Politiker aus dem bürgerlichen Lager detaillierte Gedanken über eine Einwanderungspolitik machten. Es war über Jahre für deutsche Politiker nicht opportun, öffentlich von einem Zuwanderungsland zu sprechen. Erst mit der Reform des Staatsangehörigkeitsrechts und der erleichterten Einbürgerung in den Jahren nach der Jahrtausendwende setzte ein Umdenken ein.

Die Einführung der sog. Green Card für ausländische IT-Spezialisten im Jahr 2000 unterstützte diesen Prozess. Mit dem Inkrafttreten des neuen Zuwanderungsgesetzes von 2005 wurde auch für Deutschland der Gebrauch der Bezeichnung „Zuwanderungsland“ geläufig. Mit dem Nationalen Integrationsplan von 2007 war endgültig die Basis einer sich zu einem Zuwanderungsland bekennenden Integrationspolitik geschaffen, und Deutschland bekannte sich zu einem Einwanderungsland.

Die heutige emotionale Gefühlslage vieler Migranten in Deutschland verdeutlicht am besten folgendes Zitat:

Über zwei Drittel der Zuwanderer (69 Prozent) in Deutschland fühlen sich wohl. Die meisten empfinden sich überwiegend als Teil der deutschen Gesellschaft (58 Prozent), nur fünf Prozent fühlen sich überhaupt nicht als Teil der deutschen Gesellschaft. [...] Die meisten Zuwanderer empfinden diese doppelte Verbundenheit als Vorteil und empfinden keinen Identitätskonflikt. Drei Viertel von ihnen möchten die Werte und Traditionen aus der Herkunftsgesellschaft mit Werten und Traditionen in Deutschland verbinden. ${ }^{5}$

Diese Gefühlslage wurde 2011 intensiv durch Äußerungen sowie Publikationen des ehemaligen Vorstandsmitglieds der Bundesbank, Thilo Sarrazin, irritiert. Eine sachliche Auseinandersetzung mit diesem Thema wurde dadurch

\footnotetext{
${ }^{4}$ Vgl. Statistisches Bundesamt, 2012/1.

${ }^{5}$ Bertelsmann Stiftung Gütersloh 2009, S. 4.
} 
erschwert, weil die undifferenzierte Betrachtung von Sarrazin auf fruchtbaren Boden fiel und mit der Frage nach der Integrationsbereitschaft von Migranten verbunden wurde. In der Öffentlichkeit wurde nunmehr pauschal über Integrationsunwilligkeit und Integrationsunfähigkeit einzelner Migrantengruppen, insbesondere von Muslimen, gesprochen. ${ }^{6}$ Dass sich die tatsächliche Situation allerdings deutlich differenzierter und in Abhängigkeit der Integrationspolitik darstellt, ist mit den Aussagen von Kober zu belegen. „Je länger also jemand in Deutschland lebt, umso mehr fühlt er sich hier als Teil der Gesellschaft: Die emotionale Verwurzelung in Deutschland nimmt mit der Dauer des Aufenthalts zu. ${ }^{\text {"7 }}$

\section{Bevölkerung/Migrantenbevölkerung in Deutschland 2012}

Die bisherigen Ausführungen werden in diesem sowie weiteren Kapiteln durch statistische Angaben untermauert:

\begin{tabular}{|l|r|r|r|}
\hline $\begin{array}{l}\text { Herkunft } \\
\text { (Stand 31.12.2011) }\end{array}$ & Personenanzahl & $\begin{array}{c}\text { Anteil an der auslän- } \\
\text { dischen Bevölkerung }\end{array}$ & $\begin{array}{c}\text { Anteil an der } \\
\text { Gesamtbevölkerung }\end{array}$ \\
\hline Gesamtbevölkerung & 81.754 .000 & & $100 \%$ \\
\hline Deutsche ohne MH & 65.792 .000 & & $80,5 \%$ \\
\hline Deutsche mit MH & 15.962 .000 & & $19,5 \%$ \\
\hline Afghanistan & 56.563 & $0,82 \%$ & $0,07 \%$ \\
\hline Libanon & 35.029 & $0,50 \%$ & $0,04 \%$ \\
\hline Irak & 82.438 & $1,19 \%$ & $0,10 \%$ \\
\hline Iran & 53.920 & $0,78 \%$ & $0,07 \%$ \\
\hline Marokko & 63.037 & $0,91 \%$ & $0,08 \%$ \\
\hline Türkei & 1.607 .161 & $23,19 \%$ & $1,97 \%$ \\
\hline $\begin{array}{l}\text { Alle ausländischen } \\
\text { Staatsangehörigen }\end{array}$ & 7.369 .900 & $100 \%$ & $9,01 \%$ \\
\hline
\end{tabular}

Tab. 1: Bevölkerung nach ausgewählten Staatsangehörigkeiten und Herkünften ${ }^{8}$

Bei den Diskussionen wurde oft die gesamte Migrantengruppe als eine homogene Einheit betrachtet, Unterschiede nach Herkunft, Ethnie, Bildungssituation oder Alter wurden dabei nicht regelmäßig einbezogen. Dabei sind die Lebenssituationen der Migranten sehr vielfältig und vielschichtig. An dieser Stelle

\footnotetext{
${ }^{6}$ Vgl. Berlin-Institut für Bevölkerung und Entwicklung 2009.

${ }^{7}$ Kober 2001, S. 13.

${ }^{8}$ Vgl. Statistisches Bundesamt 2012/1 und 2012/5, eigene Berechnung.
} 
soll nicht auf diese Details eingegangen werden, das hat die Heidelberger Forschungsgruppe Sociovision bereits 2008 mit der ersten Studie über die Lebensumstände und die Milieus der Migranten vorgenommen. ${ }^{9}$

\section{Demografie der Bevölkerung}

\begin{tabular}{|l|r|r|r|r|}
\hline $\begin{array}{l}\text { Bevölkerung in 1.000 } \\
- \text { im Alter von ... bis unter ... Jahren }\end{array}$ & Gesamt & Deutsch & mit MH & $\begin{array}{c}\text { mit türkischem } \\
\text { MH }\end{array}$ \\
\hline $00-05$. & 3.288 & 2.140 & 1.148 & 220 \\
\hline $05-10$. & 3.453 & 2.323 & 1.130 & 235 \\
\hline $10-15$ & 3.846 & 2.712 & 1.134 & 270 \\
\hline $15-20$ & 4.147 & 3.022 & 1.125 & 274 \\
\hline $20-25$ & 4.972 & 3.859 & 1.112 & 233 \\
\hline $25-35$ & 9.845 & 7.372 & 2.473 & 454 \\
\hline $35-45$ & 11.439 & 8.889 & 2.550 & 538 \\
\hline $45-55$ & 13.227 & 11.062 & 2.166 & 350 \\
\hline $55-65$ & 10.416 & 8.801 & 1.614 & 198 \\
\hline $65-75$ & 9.409 & 8.465 & 944 & 156 \\
\hline $75-85$ & 5.839 & 5.379 & 460 & 29 \\
\hline $85-95$ & 1.764 & 1.662 & 102 & - \\
\hline 95 und mehr & 109 & 105 & - & - \\
\hline Insgesamt & $\mathbf{8 1 . 7 5 4}$ & $\mathbf{6 5 . 7 9 2}$ & $\mathbf{1 5 . 9 6 2}$ & $\mathbf{2 . 9 5 6}$ \\
\hline
\end{tabular}

Tab. 2: Demografische Struktur in Deutschland nach ausgewählten Bevölkerungsgruppen ${ }^{10}$

Das Statistische Bundesamt gibt die Zahl der gegenwärtig in Deutschland lebenden Menschen mit MH mit 15,9 Millionen an. Das entspricht einem Bevölkerungsanteil von 19,5\%, d. h. jede fünfte in Deutschland lebende Person hat einen MH. Der Anteil der Bevölkerung mit türkischem MH liegt bei 3,6\% im Verhältnis zur Gesamtbevölkerung. 3,2 Millionen Personen mit MH sind über 55 Jahre alt. Der Anteil der älteren Personen macht dabei 19,5\% der Migranten aus. Bei der türkischstämmigen Bevölkerung haben 383.000 Personen von 2,9 Millionen dieses Alter überschritten, dort liegt der Anteil bei 13,2\%. D. h., die Bevölkerung mit $\mathrm{MH}$ im Allgemeinen und die türkischstämmige Bevölkerung im Speziellen sind deutlich jünger als die deutsche, bei der der Anteil der über 55-Jährigen $37,1 \%$ beträgt. Die Zahl der unter 20-Jährigen beträgt bei den Menschen mit MH 4,5 Millionen Das entspricht einem Anteil von 28,4\%.

\footnotetext{
${ }^{9}$ Vgl. Sinus Sociovision 2008.

${ }^{10}$ Vgl. Statistisches Bundesamt 2012/5, eigene Darstellung.
} 
Bei der türkischstämmigen Bevölkerung liegt dieser Anteil bei 33,8\%. Im Vergleich dazu sind lediglich 15,5\% der deutschen Bevölkerung unter 20 Jahre alt. ${ }^{11}$ Für den Arbeitsmarkt und für unsere Sozialsysteme sind diese Zahlen von großer Bedeutung. Sie liefern die Grundlagen für künftige Maßnahmen für den Arbeitsmarkt sowie die Rentensicherung.

Einen Kurzüberblick gibt die Tabelle 2 über das durchschnittliche Alter der Bevölkerung nach ausgewählten Kriterien. Die Differenz zwischen der deutschen Bevölkerungsgruppe und den Migranten beträgt 10,9 Jahre. Das Durchschnittsalter der türkischstämmigen Gruppe ist im Vergleich zu dem der Migranten noch einmal um 3,7 Jahre niedriger. Lediglich bei Migranten, die aus Afrika stammen, liegt das Durchschnittsalter mit 28,7 Jahren und denen aus Südund Südostasien mit 31,4 Jahren unter dem der türkischstämmigen Migranten. Diese Bevölkerungsgruppen machen zusammen allerdings lediglich einen Anteil von 8,2\% unter den Migranten aus. Das Durchschnittsalter der Bevölkerung liegt bei 44,0 Jahren, das der deutschen bei 46,1, das der Migranten bei 35,2 und der türkischstämmigen Migranten bei 31,5 Jahren. ${ }^{12}$ Der demografische Wandel, der eine erhebliche Rolle bei Planungen zur Zukunft von Gesellschaften spielt, wird anhand der oben aufgeführten Zahlen verdeutlicht.

\section{Bildungssituation der Bevölkerung}

Das Bildungsniveau der Migranten ist durchschnittlich schlechter als das der Deutschen: 21,6\% der türkischen Migranten verlassen ohne Schulabschluss die Schule. Dieser Anteil liegt bei der deutschen Bevölkerung bei 1,5\%. Der Anteil der Bevölkerung ohne Berufsabschluss liegt bei den Personen mit türkischen MH bei 40,8\% und bei den deutschen bei $12,8 \%$.

\section{Schülerinnen/Schüler nach Schulformen}

Nachfolgend werden Daten zu den Bereichen Bildung, Einbürgerungen, von Wanderungsbewegungen und von Lebensstilen sowie der Erwerbstätigkeit von Migranten in Deutschland dargelegt.

\footnotetext{
${ }^{11}$ Vgl. ebenda, eigene Berechnungen.

${ }^{12}$ Vgl. Statistisches Bundesamt 2012/5, eigene Berechnungen.
} 


\begin{tabular}{|c|c|c|c|c|}
\hline $\begin{array}{l}\text { Allgemeiner Schulabschluss } \\
\text { (Angaben in 1.000) }\end{array}$ & Gesamt & $\begin{array}{c}\text { Deutsche } \\
\text { Bevölkerung }\end{array}$ & $\begin{array}{l}\text { Bevölkerung } \\
\text { mit } \mathrm{MH}\end{array}$ & $\begin{array}{c}\text { Bevölkerung } \\
\text { mit türkischem } \\
\text { MH }\end{array}$ \\
\hline Haupt-(Volks-)schulabschluss & 25.854 & 21.871 & 3.983 & 797 \\
\hline Abschluss Polytechnischen Oberschule der DDR & 5.027 & 4.934 & 93 & - \\
\hline Realschul- oder gleichwertiger Abschluss & 15.479 & 12.985 & 2.494 & 318 \\
\hline Fachhochschulreife & 4.527 & 3.851 & 676 & 82 \\
\hline Abitur & 14.430 & 11.658 & 2.772 & 203 \\
\hline Ohne Schulabschluss & 2.684 & 1.019 & 1.665 & 639 \\
\hline Noch in Ausbildung/noch nicht schulpflichtig & 13.302 & 9.116 & 4.186 & 906 \\
\hline \multicolumn{5}{|l|}{ Beruflicher Abschluss } \\
\hline Mit berufsqualifizierendem Abschluss & 51.535 & 45.112 & 6.423 & 640 \\
\hline Lehre/Berufsausbildung im dualen System & 32.981 & 29.142 & 3.838 & 485 \\
\hline Berufsfachschule & 1.667 & 1.393 & 274 & 22 \\
\hline Meister/Techniker/Fachschulabschluss & 5.401 & 4.904 & 497 & 44 \\
\hline Berufsakademie & 863 & 721 & 141 & k. A. \\
\hline Fachhochschulabschluss & 3.489 & 3.064 & 426 & 24 \\
\hline Universitätsabschluss/Promotion & 5.907 & 4.806 & 1.101 & 53 \\
\hline Ohne berufsqualifizierenden Abschluss & 29.817 & 20.348 & 9.469 & 2.310 \\
\hline Noch in schulischer Ausbild./nicht schulpflichtig & 13.255 & 9.081 & 4.174 & 904 \\
\hline Noch in berufsqualifizierender Ausbildung & 3.537 & 2.676 & 860 & 165 \\
\hline Ohne Abschluss & 12.575 & 8.312 & 4.264 & 1.206 \\
\hline
\end{tabular}

Tab. 3: Bildungsniveau der Bevölkerung in Deutschland. ${ }^{13}$

Seit dem Inkrafttreten des neuen Staatsangehörigkeitsgesetzes erhalten Kinder mit der Geburt bei entsprechender Voraussetzung die deutsche Staatsbürgerschaft. Sie werden als deutsche Staatsbürger geführt. Die Statistik erfasst die Schüler in diesem Bereich nur nach Staatsangehörigkeit.

Der Anteil der Schüler mit türkischer Staatsangehörigkeit unter den ausländischen Schülern beträgt 37,2 \%. Der Anteil bei den Hauptschülern liegt bei 44,6\%, bei den Realschülern liegt er etwa gleich bei 45,0\% und bei den Gymnasiasten bei $28,8 \%{ }^{14}$

\section{Einbürgerungen in Deutschland}

Wenn man als Indikator der Integration auch die Einbürgerung berücksichtigen will, sollten die nachfolgenden Zahlen aus der Tabelle ebenfalls betrachtet werden. Zwischen 1981 bis 1999 wurden insgesamt 2.672.676 Personen in

\footnotetext{
${ }^{13}$ Vgl. ebenda, eigene Darstellung.

${ }^{14}$ Vgl. Statistisches Bundesamt, 2011/3.
} 
Deutschland eingebürgert. ${ }^{15}$ Davon waren 281.000 Personen mit türkischer Abstammung. Die Einbürgerungszahlen waren seit Änderung des Staatsangehörigkeitsrechts sinkend, seit 2008 steigen sie wieder geringfügig an. Allerdings ist anzumerken, dass über die Hälfte der Einbürgerungen seit 2008 unter Hinnahme von Mehrstaatigkeit durchgeführt wurden. ${ }^{16}$

\begin{tabular}{|l|r|r|r|}
\hline & $\begin{array}{r}\text { Schüler/innen } \\
\text { insgesamt }\end{array}$ & $\begin{array}{r}\text { deutsche } \\
\text { Schüler/innen }\end{array}$ & $\begin{array}{r}\text { ausländische } \\
\text { Schüler/innen }\end{array}$ \\
\hline Vorklassen & 9.580 & 8.689 & 8.91 \\
\hline Schulkindergärten & 19.153 & 16.383 & 2.770 \\
\hline Grundschulen & 2.837 .737 & 262.2676 & 215.061 \\
\hline Schulartunabhängige Orientierungsstufe & 111.199 & 99.048 & 12.151 \\
\hline Hauptschulen & 703.525 & 566.795 & 136.730 \\
\hline Schularten mit mehreren Bildungsgängen & 370.852 & 351.675 & 19.177 \\
\hline Realschulen & 1.166 .509 & 1.069 .899 & 96.610 \\
\hline Gymnasien & 2.475 .174 & 2.365 .994 & 109.180 \\
\hline Integrierte Gesamtschulen & 584.770 & 509.967 & 74.803 \\
\hline Freie Waldorfschulen & 80.843 & 79.340 & 1.503 \\
\hline Förderschulen & 377.922 & 328.783 & 49.139 \\
\hline Abendhauptschulen & 1.100 & 711 & 389 \\
\hline Abendrealschulen & 21.345 & 16.616 & 4.729 \\
\hline Abendgymnasien & 19.155 & 16.449 & 2.706 \\
\hline Kollegs & 18.030 & 16.839 & 1.191 \\
\hline Insgesamt & $\mathbf{8 . 7 9 6 . 8 9 4}$ & $\mathbf{8 . 0 6 9 . 8 6 4}$ & $\mathbf{7 2 7 . 0 3 0}$ \\
\hline
\end{tabular}

Tab. 4: Schüler/innen (Deutsche, Ausländer) nach Schularten im Schuljahr 2010/2011 ${ }^{17}$

\begin{tabular}{|l|r|r|r|r|r|r|r|}
\hline Jahr & Afghanistan & Libanon & Irak & Iran & Marokko & Türkei & Gesamt \\
\hline 2000 & 4.773 & 5.673 & 984 & 14.410 & 5.008 & 82.861 & 186.688 \\
\hline 2001 & 5.111 & 4.486 & 1.264 & 12.020 & 4.425 & 76.573 & 178.098 \\
\hline 2002 & 4.750 & 3.300 & 1.721 & 8.375 & 3.800 & 64.631 & 154.547 \\
\hline 2003 & 4.948 & 2.651 & 2.999 & 5.504 & 4.118 & 56.244 & 140.731 \\
\hline 2004 & 4.077 & 2.265 & 3.564 & 3.539 & 3.820 & 44.465 & 127.153 \\
\hline 2005 & 3.133 & 1.969 & 4.163 & 8.824 & 3.684 & 32.661 & 117.241 \\
\hline 2006 & 3.063 & 2.030 & 3.693 & 12.601 & 3.546 & 33.388 & 124.566 \\
\hline 2007 & 2.831 & 1.754 & 4.102 & 10.458 & 3.489 & 28.861 & 113.030 \\
\hline 2008 & 2.512 & 1.675 & 4.229 & 6.903 & 3.130 & 24.449 & 94.470 \\
\hline 2009 & 3.549 & 1.759 & 5.136 & 5.732 & 3.042 & 24.647 & 96.122 \\
\hline 2010 & 3.520 & 1.697 & 5.228 & 6.522 & 2.806 & 26.192 & 101.570 \\
\hline 2011 & 2.711 & 1.433 & 4.790 & 2.728 & 3.011 & 28.103 & 106.897 \\
\hline Summe & $\mathbf{4 4 . 9 7 8}$ & $\mathbf{3 0 . 6 9 2}$ & $\mathbf{4 1 . 8 7 3}$ & $\mathbf{9 7 . 6 1 6}$ & $\mathbf{4 3 . 8 7 9}$ & $\mathbf{5 2 3 . 0 7 5}$ & $\mathbf{1 . 5 4 1 . 1 1 3}$ \\
\hline
\end{tabular}

Tab. 5: Einbürgerungen 2000-2011 nach ausgewählten Herkunftsländern ${ }^{18}$

\footnotetext{
${ }^{15}$ Vgl. Ebenda.

${ }^{16}$ Vgl. Statistisches Bundesamt, 2012/5.

${ }^{17}$ Vgl. Statistisches Bundesamt, 2011/1, Statistisches Bundesamt, 2011/3
} 


\section{Zuzüge und Fortzüge nach Deutschland}

Im Jahr 2011 kamen 958.000 Personen nach Deutschland und 679.000 Personen wanderten aus. Gegenüber dem Vorjahr hat sich damit die Zahl der Zuzüge um 160.000 erhöht, während die Zahl der Fortzüge lediglich um 8.000 anstieg. Für 2011 ergibt sich mithin ein Wanderungsüberschuss von 279.000 Personen. Ähnlich hoch war der Wanderungssaldo zuletzt im Jahr 2001 mit 273.000 Personen. ${ }^{19}$ Der Wanderungssaldo zwischen 1991 und 2010 für Ausländer betrug kumuliert 2,9 Millionen Personen. ${ }^{20}$

\begin{tabular}{|c|c|c|r|}
\hline $\begin{array}{c}\text { Deutschland } \\
\text { Jahr }\end{array}$ & $\begin{array}{c}\text { Zuzüge } \\
\text { Personen }\end{array}$ & $\begin{array}{c}\text { Fortzüge } \\
\text { Personen }\end{array}$ & $\begin{array}{c}\text { Saldo } \\
\text { Personen }\end{array}$ \\
\hline 2000 & 841.158 & 674.038 & 167.120 \\
\hline 2001 & 879.217 & 606.494 & 272.723 \\
\hline 2002 & 842.543 & 623.255 & 219.288 \\
\hline 2003 & 768.975 & 626.330 & 142.645 \\
\hline 2004 & 780.175 & 697.633 & 82.542 \\
\hline 2005 & 707.352 & 628.399 & 78.953 \\
\hline 2006 & 661.855 & 639.064 & 22.791 \\
\hline 2007 & 680.766 & 636.854 & 43.912 \\
\hline 2008 & 682.146 & 737.889 & -55.743 \\
\hline 2009 & 721.014 & 733.796 & -12.782 \\
\hline 2010 & 798.282 & 670.605 & 127.677 \\
\hline
\end{tabular}

Tab. 6: Wanderungen zwischen Deutschland und dem Ausland ${ }^{21}$

\section{Haushalte und Lebenslagen der Bevölkerung}

Die durchschnittliche Haushaltsgröße der Personen mit MH ist im Vergleich zu der deutschen Bevölkerung mit 2,4 Personen gegenüber 2,0 Personen um 0,4 höher. Zudem leben Migranten seltener allein als die deutsche Bevölkerung, 14,8\% gegenüber 23,1\%. Der Anteil Alleinlebender bei der türkischstämmigen Bevölkerung liegt bei 8,0\%. Die durchschnittliche Zahl der Kinder liegt bei den Personen mit MH um 0,2 höher bei 1,8 als bei der deutschen Bevölkerung.

Ehepaare ohne Kinder, Alleinerziehende oder alternative Lebensformen sind bei den Migranten dagegen erheblich seltener. 2,1\% der deutschen Bevölkerung

\footnotetext{
${ }^{18}$ Vgl. Statistisches Bundesamt, 2012/6, verschiedene Jahrgänge, eigene Berechnung.

${ }^{19}$ Vgl. Statistisches Bundesamt, 2012/3, S. 618.

${ }^{20}$ Vgl. Statistisches Bundesamt, 2012/4.

21 Vgl. ebenda.
} 
leben in Lebensgemeinschaften. Der Anteil bei den Personen mit MH beträgt $1,2 \%$ und bei den Personen mit türkischen MH 0,5\%. Weitere Differenzierungen ergeben, dass die Haushalte mit türkischem $\mathrm{MH}$ durchschnittlich mit 3,5 Personen die meisten Mitglieder umfassen. ${ }^{22}$

\begin{tabular}{|c|c|c|c|c|c|c|c|c|}
\hline \multirow{6}{*}{$\begin{array}{l}\text { Migrationsstatus } \\
\text { der Bezugsperson } \\
\text { und der Mitglieder } \\
\text { der Lebensform }\end{array}$} & \multicolumn{8}{|c|}{ Lebensformen } \\
\hline & \multirow{4}{*}{$\begin{array}{l}\text { insge- } \\
\text { samt }\end{array}$} & \multirow{4}{*}{$\begin{array}{l}\text { Paare } \\
\text { ohne } \\
\text { Kinder }\end{array}$} & \multirow{4}{*}{$\begin{array}{l}\text { Allein- } \\
\text { stehen- } \\
\text { de }\end{array}$} & \multicolumn{4}{|c|}{ Familien } & \multirow{5}{*}{$\begin{array}{l}\text { Zahl der } \\
\text { Kinder } \\
\text { im } \\
\text { Schnitt }\end{array}$} \\
\hline & & & & \multirow{3}{*}{$\begin{array}{l}\text { Famili- } \\
\text { en ins- } \\
\text { gesamt }\end{array}$} & \multicolumn{3}{|c|}{ davon mit } & \\
\hline & & & & & 1 & 2 & $\begin{array}{l}\text { und } \\
\text { mehr }\end{array}$ & \\
\hline & & & & & \multicolumn{3}{|c|}{ Kindern } & \\
\hline & \multicolumn{7}{|c|}{ Angaben in 1.000} & \\
\hline $\begin{array}{l}\text { Lebensformen ins- } \\
\text { gesamt }\end{array}$ & 41.099 & 11.783 & 17.607 & 11.709 & 6.144 & 4.205 & 1.360 & 1,6 \\
\hline Person ohne MH & 34.557 & 10.284 & 15.239 & 9.034 & 4.961 & 3.200 & 874 & 1,6 \\
\hline Personen mit MH & 6.542 & 1.500 & 2.367 & 2.675 & 1.184 & 1.005 & 487 & 1,8 \\
\hline $\begin{array}{l}\text { Personen mit tür- } \\
\text { kischem } \mathrm{MH}\end{array}$ & 1.035 & 183 & 239 & 614 & 201 & 247 & 166 & 2,1 \\
\hline
\end{tabular}

Tab. 7: Lebensformen der Bevölkerung ${ }^{23}$

\section{Erwerbssituation der Bevölkerung}

Der Anteil der Erwerbspersonen bei der deutschen Bevölkerung liegt im Vergleich geringfügig höher als bei den Migranten: 52,5\% der deutschen Bevölkerung sind Erwerbspersonen, bei den Migranten liegt dieser Anteil bei 48,8\%, und bei Personen mit türkischen MH liegt der Anteil bei 43,1\%.

\begin{tabular}{|l|c|c|c|}
\hline (Angaben in 1.000) & Erwerbspersonen & Erwerbstätige & Erwerbslose \\
\hline Gesamtbevölkerung & 42.372 & 39.869 & 2.504 \\
\hline Deutsche & 34.576 & 32.813 & 1.763 \\
\hline Personen mit MH & 7.797 & 7.056 & 741 \\
\hline Personen mit türkischem MH & 1.273 & 1.104 & 169 \\
\hline
\end{tabular}

Tab. 8: Angaben zur Erwerbssituation der Bevölkerung ${ }^{24}$

Der Anteil der Erwerbslosen an der Gesamtbevölkerung beträgt 5,9\%, in der deutschen Bevölkerung bei 5,1\%. Bei den Migranten ist dieser Anteil bei den

\footnotetext{
${ }^{22}$ Vgl. ebenda, eigene Berechnung,

23 Vgl. Statistisches Bundesamt, 2012/4.

${ }^{24}$ Vgl. Statistisches Bundesamt, 2012/5, eigene Berechnungen.
} 
Bevölkerungsgruppen nach Herkünften sehr unterschiedlich, durchschnittlich liegt die Quote jedoch bei 9,5\%, bei der türkischstämmigen Bevölkerung liegt sie bei $13,3 \%$.

\begin{tabular}{|l|c|c|c|c|c|}
\hline & $\begin{array}{c}\text { Anzahl der Er- } \\
\text { werbspersonen } \\
\text { (Angaben in } \\
\text { 1.000) }\end{array}$ & $\begin{array}{c}\text { Anteil am Pro- } \\
\text { duzierenden } \\
\text { Gewerbe }\end{array}$ & $\begin{array}{c}\text { Anteil am } \\
\text { Handel/ } \\
\text { Gastgewerbe }\end{array}$ & $\begin{array}{c}\text { Anteil an } \\
\text { sonstigen } \\
\text { Dienstleistun- } \\
\text { gen }\end{array}$ & $\begin{array}{c}\text { Anteil an der } \\
\text { Landwirtschaft }\end{array}$ \\
\hline Gesamtbevölkerung & 39.869 & $28,2 \%$ & $25,2 \%$ & $44,9 \%$ & $1,7 \%$ \\
\hline Personen ohne $\mathrm{MH}$ & 65.792 & $27,4 \%$ & $24,1 \%$ & $46,6 \%$ & $1,9 \%$ \\
\hline Personen mit $\mathrm{MH}$ & 15.962 & $32,0 \%$ & $30,4 \%$ & $37,0 \%$ & $0,6 \%$ \\
\hline Personen mit türkischem $\mathrm{MH}$ & 2.956 & $36,3 \%$ & $33,0 \%$ & $30,4 \%$ & - \\
\hline
\end{tabular}

Tab. 9: Angaben der Erwerbspersonen nach Wirtschaftsbereichen ${ }^{25}$

Tabelle 9 gibt eine Übersicht über die Verteilung der Erwerbspersonen nach Wirtschaftsbereichen. Der Anteil der Personen mit türkischem MH, die im produzierenden Gewerbe und im Handel/Gastgewerbe tätig ist, liegt höher als der der deutschen Bevölkerung, und sie ist auch höher als bei Personen mit MH und anderen Herkünften als der Türkei. Der Anteil in den sonstigen Dienstleistungen liegt dagegen deutlich unter dem der o. g. Gruppen. Eine der möglichen Erklärungen dafür ist, dass die Gruppe der türkischstämmigen Personen als ehemalige Gastarbeiter hauptsächlich in den Bereichen ungelernter Arbeit tätig waren und weiterhin sind, denn ursprünglich waren ja viele Gastarbeiter für körperlich schwere Arbeiten eingestellt worden. Weitere Gründe mögen in den persönlichen (Bildungs-)Voraussetzungen liegen.

\section{Einkommenssituation der Bevölkerung}

In den unteren Einkommensgruppen ist der Anteil von Migranten hoch. Der Anteil derjenigen, die bis 1.500,- Euro netto verdienen, liegt bei den Deutschen bei $34,7 \%$, bei den Migranten dagegen bei ca. $40 \%$. Bei der Einkommensklasse mit über 4.500,- Euro zeigt sich ebenfalls eine Disparität: Der Anteil der deutschen Bevölkerung liegt bei 9,2\% und bei den Migranten bei 5,2\%. Die Gründe dafür sind u. a. dem Bildungsstand der Bevölkerung zu entnehmen.

\footnotetext{
${ }^{25}$ Vgl. ebenda, eigene Berechnungen, Aufrundung der Angaben auf eine Stelle hinter dem Komma.
} 


\section{Die Ethnische Ökonomie}

In Deutschland ist in den letzten Jahrzehnten ein Wandel feststellbar, der die Gesellschaft nicht nur in politischen oder religiösen Fragen intensiv beschäftigt. Auch die ökonomische Dimension des gesellschaftlichen Wandels ist ein entscheidender Faktor für die Fortentwicklung einer gemeinsamen Zukunft. Oft werden Migranten als Belastung für den Arbeitsmarkt wahrgenommen. Dieses schiefe Bild in der Gesellschaft wird meistens durch die einseitige Berichterstattung der Presse gefördert. ${ }^{26}$ Die defizitorientierte Vorgehensweise und die problembasierte Darstellung der Situation verzerrt das reale Bild der Gesellschaft. Negativansätze wie z. B. fehlender Bildungserfolg, hohe Arbeitslosigkeit, Sprachprobleme und Ghettobildung sind schnell gefundene Argumentationsketten, die im Einzelfall zutreffen mögen, aber jedenfalls nicht generalisierbar sind.

\begin{tabular}{|c|c|c|c|c|}
\hline \multirow{2}{*}{$\begin{array}{l}\text { Nettoeinkommen von ... } \\
\text { bis } \\
\text { unter ... Euro im Monat }\end{array}$} & \multicolumn{4}{|c|}{ Angaben in $\mathbf{1 . 0 0 0}$} \\
\hline & Gesamt & Deutsche & mit MH & $\begin{array}{c}\text { türkischer } \\
\mathrm{MH}\end{array}$ \\
\hline unter 500 & 926 & 737 & 189 & 23 \\
\hline $500-900$ & 4.049 & 3.270 & 778 & 106 \\
\hline $900-1.300$ & 5.588 & 4.646 & 942 & 137 \\
\hline $1.300-1.500$ & 2.993 & 2.529 & 464 & 69 \\
\hline $1.500-2.000$ & 6.287 & 5.320 & 967 & 170 \\
\hline $2.000-2.600$ & 5.894 & 4.927 & 967 & 170 \\
\hline $2.600-3.200$ & 4.073 & 3.455 & 619 & 108 \\
\hline $3.200-4.500$ & 4.911 & 4.307 & 604 & 91 \\
\hline 4.500 und mehr & 3.280 & 2.972 & 308 & 31 \\
\hline
\end{tabular}

Tab. 10: Privathaushalte am Haupt- und Nebenwohnsitz mit Nettoeinkommen ${ }^{27}$

Das Statistische Bundesamt gibt die Zahl der Selbständigen mit MH zum Jahresende 2011 insgesamt mit 726.000 Personen an, davon sind 324.000 Angehörige aus EU-Staaten. Die größte Gruppe bilden die Polnischstämmigen mit 96.000 Selbständigen, ${ }^{28}$ gefolgt von Türkischstämmigen mit 92.000, Italienischstämmi-

\footnotetext{
${ }^{26}$ Vgl. die Beiträge von Sabine Schiffer (S. 121-137) und Nina Kalwa (S. 139-158) in diesem Sammelband.

${ }^{27}$ Vgl. Statistisches Bundesamt, 2012/5.

${ }^{28}$ Anmerkung d. V.: Die Selbständigen aus Polen und der Russischen Föderation wurden in den Tabellen 11 und 12 nicht berücksichtigt. Es existieren keine relevanten Vergleichszahlen zu früheren Jahren. Die Selbständigenzahl aus diesen Ländern ist erst in den letzten Jahren auf ein nennenswertes Niveau angestiegen.
} 
gen mit 51.000 sowie 35.000 Selbständigen aus der Russischen Föderation. Die Selbständigenquote bei den Migranten liegt bei 9,3\%, und zum Vergleich: die der deutschen Bevölkerung liegt bei $10,4 \%$. Es ist davon auszugehen, dass in den kommenden Jahren der Anteil der Selbständigen in Deutschland im Rahmen des Rechts auf Freizügigkeit durch Zuzüge aus EU-Ländern weiter steigen wird. ${ }^{29}$ Verlässliche aktuelle Angaben zum Umsatz der Selbständigen mit MH konnten nicht ermittelt werden. Bisher herausgegebene Zahlen waren grobe Schätzungen, die nicht unbedingt belastbar waren.

\begin{tabular}{|l|c|c|c|c|}
\hline \multirow{2}{*}{ Stand 31.12.2011) } & \multicolumn{3}{|c|}{ Angaben zur Herkunft } \\
\cline { 2 - 5 } & Personen mit MH & griechisch & italienisch & türkisch \\
\hline Selbständige 2012 & 726.000 & 25.000 & 51.000 & 92.000 \\
\hline Selbständige 2004 & $\begin{array}{c}286.000 \\
\text { (nur Ausländer) }\end{array}$ & 27.500 & 49.500 & 60.500 \\
\hline Selbständige 1991 & $\begin{array}{c}175.000 \\
\text { (nur Ausländer) }\end{array}$ & 22.500 & 33.500 & 39.500 \\
\hline Anteil an den Selbständigen mit MH 2012 & & $3,4 \%$ & $7,0 \%$ & $12,6 \%$ \\
\hline Selbständigenquote 2012 & $9,3 \%$ & $11,9 \%$ & $11,7 \%$ & $7,5 \%$ \\
\hline
\end{tabular}

Tab. 11: Entwicklung der Selbständigkeit von ausgewählten Migrantengruppen. ${ }^{30}$

\section{Biographien von Migranten aus islamisch geprägten Herkunftsstaaten}

Als Beispiel-Biografien für erfolgreiche Migranten aus islamisch geprägten Herkunftsstaaten wurden drei Interviews geführt und in Fließtext wiedergegeben. Die Angaben zu den Personen wurden anonymisiert, Familiennamen weggelassen und Vornamen geändert.

\subsection{Biographie Selin (w, Herkunftsstaat Türkei)}

Selin ist 42 Jahre alt und von Beruf Kinderkrankenschwester. Sie stammt ursprünglich aus dem nördlichen Bereich Zentralanatoliens, ist verheiratet, hat zwei Töchter und einen Sohn. Selin reiste mit vier Jahren als Tochter einer sog. Gastarbeiterfamilie nach Deutschland ein. Sie hat noch einen älteren und einen jüngeren Bruder. Ihre Mutter ist 1971 als Gastarbeiterin nach Deutschland gekommen. Erst ein Jahr später zog der Vater, ebenfalls als Gastarbeiter, nach.

\footnotetext{
${ }^{29}$ Vgl. Statistisches Bundesamt, 2012/5, eigene Berechnungen.

${ }^{30} \mathrm{Vgl}$. ebenda, ifm 2005, eigene Berechnungen.
} 
Selin konnte keinen Kindergarten besuchen. Bedingt durch die Berufstätigkeit der Eltern musste sie auf den kleinen Bruder aufpassen. Der ältere Bruder kam erst mit 12 Jahren nach Deutschland. Bis dahin lebte er, wie sie früher auch, bei den Großeltern in der Türkei. Der ältere Bruder konnte in Deutschland nur die Hauptschule besuchen. Er arbeitet heute als Arbeitnehmer in der Metallbranche. Der jüngere Bruder ist als Selbständiger in der Baubranche tätig. Selin wurde 1977 eingeschult. Sie lernte die deutsche Sprache auf der Straße. Es gab keinen Sprachkurs, den sie besuchen konnte. Sie hatte Kontakt zu der Enkelin des deutschen Vermieters. Der Kontakt mit ihr und anderen Kindern war die „Sprachschule“ für sie.

Selin hatte zwei gute deutsche Freundinnen, die ihr Leben beeinflusst haben. „Ich bin selten zu meinen Freundinnen gegangen. Das hatte mit gesellschaftlichen Zwängen, aber auch mit Traditionen zu tun. Es war zu der Zeit nicht üblich, dass deutsche Kinder in unsere Familien zu Besuch kamen. Es war eher eine Ausnahme, wenn ein türkisches Mädchen die Freundinnen zu Hause besuchte und die deutschen Freundinnen in die türkische Familie kamen. Selbstverständlich gab es Unterschiede in den Lebensweisen, angefangen beim Essen bis hin zur Musikkultur. Das mag ein Grund gewesen sein, warum der Kontakt relativ gering war."

Selins Freundinnen haben es auch im Berufsleben geschafft. Eine Freundin ist heute Modedesignerin. Sie sagt, sie hätte es von sich selbst auch nicht erwartet, doch auch sie schaffte es durch Fleiß und Engagement.

Nach Abschluss der Realschule besuchte Selin die Berufsfachschule. Als sie mit 18 Jahren auf eigenen Wunsch heiratete, brach sie die 12. Klasse ab. Sie sagt: „Eigentlich wollte ich meine schulische Ausbildung weitermachen und hatte Interesse an einem Studium. Am besten wäre ein Medizinstudium gewesen. Meine Lehrerin empfahl mir bereits in der zehnten Klasse eine Ausbildung als Arzthelferin oder Verkäuferin zu machen. Ich wollte auf jeden Fall eine Berufsausbildung machen und damit meine finanzielle Unabhängigkeit herstellen. Ich wollte jedoch meine Schulbildung soweit es geht weiterführen. Deshalb entschied ich mich zunächst für den Weiterbesuch der 11. Klasse.“

Durch die Heirat musste Selin ihre berufliche Zukunft neu überdenken. Bis 1990 jobbte sie als Aushilfe. Ihr Mann studierte damals und arbeitete ebenfalls 
nebenbei. „Mein Mann und ich hatten uns in den Kopf gesetzt, unsere Zukunft mit eigenen Mittel aufzubauen. Deshalb begann ich 1990 eine Ausbildung an der Kinderkrankenpflegeschule. Durch meine Schwangerschaft musste ich jedoch diese Ausbildung unterbrechen. Ein Jahr nach der Geburt unserer Zwillinge 1992 nahm ich wieder die Ausbildung an der Kinderkrankenpflegeschule auf.“

1995 beendete Selin ihre Ausbildung zur Kinderkrankenschwester erfolgreich. Sie erfuhr von ihrer Herkunftsfamilie keine Unterstützung. Während ihrer Berufstätigkeit versorgte sie ihre Kinder mit Hilfe einer Tagesmutter. Sie wollte sich beruflich weiter entwickeln. Das Ziel war ein Studium. Gesundheitliche Probleme ihrer Kinder verhinderten jedoch dieses Vorhaben. So arbeitete sie in ihrem Beruf weiter.

„Am Anfang meiner Tätigkeit war es nicht selbstverständlich, dass ich als türkische Migrantin genauso wie meine deutschen Kollegen behandelt wurde. Unangenehme Arbeiten, Nachtschichten oder Wochenendarbeiten waren eher für uns Migranten bestimmt. Das relativierte sich aber schnell. Schließlich war unsere Qualifikation genauso gut wie die unserer Kollegen," führt Selin aus. Sie berichtet, dass sie von den Vorgesetzten am Arbeitsplatz wegen ihrer Herkunft keine Diskriminierung oder Benachteiligung erfährt. Es gibt aber dennoch einzelne Kollegen, die eine negative Haltung gegenüber Migranten haben, die sie die Betroffenen auch spüren lassen.

Ihre Zufriedenheit mit ihrer Arbeit, ständige und systematische berufliche Fortbildung sowie die Arbeitsatmosphäre motivieren sie jeden Tag aufs Neue. Sie fühlt sich in ihrem Beruf und an ihrem Arbeitsplatz sehr wohl.

Es gibt weitere Arbeitskollegen mit ähnlichen Biographien.

Das gemischte Team, in dem Selin arbeitet, bestehend aus Deutschen und Migranten, empfindet sie als eine wichtige Bereicherung für sich selbst, aber auch für die Gesellschaft. Sie sagt: „Diese Vielfalt zeichnet uns in Deutschland aus. Welches Land kann schon damit aufwarten, so viele unterschiedliche Religionen, Kulturen und Traditionen in einem friedlichen Zusammenleben zu vereinen? Dieses Gut müssen wir unbedingt erhalten." Diese Vielfalt genießt Selin auch im privaten Umfeld. Ihre Nachbarn sind Deutsche, andere stammen beispielsweise aus dem Iran, aus Spanien, Marokko usw. Auch Ethnien gemischte Familien gehören zum alltäglichen Umfeld von Selin. 
„Früher waren wir die Exoten. Man fragte uns beispielsweise, ob wir Spargel kennen. Bemerkungen wie: ,Darfst Du das überhaupt?‘ gehörten zum Alltag. Heute ist alleine in der Esskultur in Deutschland die Präsenz von Migranten und ihrer Küche prägend. Ohne Pizza, Pasta, Paella, Döner, usw. ist unsere Speisekarte nicht mehr denkbar. Auf der anderen Seite tauchen der rheinische Sauerbraten, Reibekuchen, Klöße usw. auch auf den Speisekarten der Migranten auf. Wenn ich an frühere Zeiten denke, wie wir unseren deutschen Nachbarn z. B. Schafskäse, Oliven, getrocknete Tomaten, Okraschoten, Auberginen oder andere sog. exotische Lebensmittel erklären mussten, sehe ich heute, wie weit wir uns aneinander angenähert haben.“

Wenn Selin spricht, vermittelt sie den Eindruck einer disziplinierten Mutter und einer weltoffenen, erfolgreichen Frau. Sie lebt ein Leben nach ihren Vorstellungen und ermöglicht ihren Kindern all das, was ihr verwehrt geblieben ist. Sie ist ihren Kindern eine große Stütze. Beide Töchter haben eine Berufsausbildung abgeschlossen und sind berufstätig. Eine der Töchter lebt seit einem Jahr alleine im Ausland und möchte auch dort bleiben. Die Familie unterstützt die Tochter in ihrer Entscheidung.

Selin sagt dazu: „Ich dagegen habe in Deutschland mein Zuhause. Ich bin in Deutschland zufrieden. Mein sechsjähriger Sohn ist hier geboren, wächst hier auf. Er sagt: Ich bin Deutscher. Er fühlt sich auch als Deutscher. Dagegen ist unsere seelische Zufriedenheit ausbaufähig. Ich wünschte, die Gesellschaft wäre noch offener und entgegenkommender. Ich möchte mein Zuhause nicht nur als Zuhause sehen, sondern es auch als solches empfinden. Das fällt mir manchmal schwer."

Mit: „Wir gehören auch zu Deutschland!“ schließt Selin das Gespräch ab.

\subsection{Biographie Farhat (m, Herkunftsstaat Iran)}

Farhat ist 49. Er stammt aus dem Iran. Sein Vater war Beamter, seine Mutter ist Hausfrau. Er ist Sozial- und Politikwissenschaftler. Er und seine Lebensgefährtin haben einen gemeinsamen Sohn. Farhat floh 1986 vor Repressalien aus dem Iran, weil er dort um sein Leben fürchten musste. Deshalb war die Entscheidung, das Land zu verlassen, unerlässlich. Zunächst kommt er in eines der sogenannten Auffanglager für Asylsuchende. Er hatte von Anfang an großes Interesse am 
Erlernen der deutschen Sprache. Diese Möglichkeit gab es in den Unterkünften aber nicht, und er hatte damals selbst keine finanziellen Mittel, um einen privaten Sprachkurs zu besuchen. Er bemühte sich, so gut es ging, die deutsche Sprache von Freunden und aus Büchern zu lernen. Die zeitliche und räumliche Begrenzung seines Aufenthalts in Deutschland verunsicherte noch mehr, anstatt ihm mehr Sicherheit zu geben.

Farhat sagt: „Nichts war in Deutschland darauf ausgelegt, dass ich mich in Sicherheit hätte fühlen können. Im schlimmsten Fall hatte ich sogar eine Abschiebung zu befürchten. Mein Asylverfahren dauerte drei Jahre. In dieser Zeit konnte ich weder einen Sprachkurs besuchen, noch konnte ich eine Arbeit aufnehmen. Das Treffen mit anderen Flüchtlingen aus dem Iran war ebenfalls nicht möglich, weil sie in anderen Städten untergebracht waren. Erst als mein Asylverfahren abgeschlossen war, hatte ich endlich die Möglichkeit, nach vorne zu blicken. Die erste große Hürde war genommen. Nun war die Gestaltung meines täglichen Lebens unter würdigen Bedingungen an der Reihe. Mein Abitur aus dem Iran wurde nicht anerkannt, ich musste es in Deutschland noch einmal machen. ${ }^{31}$ Das bedeutete für mich weitere drei Jahre Schulbank drücken, damit ich später ein Studium aufnehmen konnte. Ich empfand die Nichtanerkennung meiner schulischen Leistungen aus dem Iran als eine Zurückweisung meiner Person. Ich hatte mir dennoch vorgenommen, trotz der Schwierigkeiten, die sich mir in den Weg stellten, diesen Weg zu gehen und zu studieren. Ich bin sehr froh, dass ich nicht aufgegeben und die Hürden genommen habe.“

Farhat arbeitet inzwischen als Sozial- und Politikwissenschaftler bei einer Landesorganisation. Auf die Frage, wie er die Situation der Zuwanderer in Deutschland künftig sieht, sagt er. „Die politische Entwicklung in Deutschland geht dahin, dass die Zuwanderer bestenfalls toleriert werden. Teile der Gesellschaft sind von der Akzeptanz der Migranten weit weg. Im Sinne einer inklusiven Gesellschaft findet kein Prozess statt. Die Mehrheitsgesellschaft unternimmt zu wenig, um die Situation zu verändern und zu verbessern. Politiker sprechen

\footnotetext{
${ }^{31}$ Hierzu eine Buchempfehlung, die die Situation von Farhat nicht als zufälligen Einzelfall, sondern als Folge institutioneller Hürden erscheinen lässt. Es ist die Geschichte dreier iranischer Brüder, die trotz aller Widerstände und gegen Abschiebungsversuche jeweils Einser-Abiture in Deutschland machten und inzwischen an Elite-Universitäten studieren: Unerwünscht. Drei Brüder aus dem Iran erzählen ihre deutsche Geschichte, Berlin 2012, 256 Seiten, 16,99€.
} 
von Vielfalt. Offensichtlich fehlt es ihnen aber an der Wahrnehmung der realen Welt der Migranten. Kritisch sind die Bereiche, in denen Politiker Zeichen setzen könnten, dieses aber nicht tun. Ein solches Zeichen wäre etwa die Installation von gesetzlichen Strukturen wie die der doppelten Staatsbürgerschaft oder kommunales Wahlrecht für alle.“

Farhat sagt weiter, dass Deutschland es mit der heutigen gesellschaftlichen und politischen Entwicklung nicht schaffen werde, tatsächlich ein Einwanderungsland zu werden. Andere Staaten, die eine offensive und angemessene Einwanderungspolitik machen, würden Deutschland abhängen. „Deutschland ist ein Land, das viele Möglichkeiten bietet. Man kann hier gut leben. Aber es erstaunt mich während meiner Auslandsaufenthalte immer wieder, wie schlecht das Image Deutschlands im Ausland ist. Es ist noch schlechter, als es in Wirklichkeit ist. Im Land sieht es in der Tat so aus, dass es sehr viele Deutsche gibt, die sich gegenüber anderen Menschen aufgeschlossen zeigen und diese kennenlernen wollen, sie sind an dem anderen sehr interessiert. Diese Bekanntschaften sind zu Freundschaften geworden. Ich möchte sie nicht missen."

Würde Farhat, falls er noch einmal aus dem Iran fliehen müsste, wieder nach Deutschland kommen? „Ich würde mir das gründlich überlegen und mir sehr intensive Gedanken machen. Ich erfahre von vielen anderen, die wegen des Studiums oder der Liebe nach Deutschland gekommen sind, dass sie bereits in der Anfangsphase ihrer Integrationsbemühungen scheitern, weil sie nicht akzeptiert werden.“

Auf die Frage nach seiner Beschreibung von Integration antwortet Farhat zum Schluss: „Die wichtigsten Indikatoren für die Integration sind die gegenseitige Neugier und der Austausch ohne Scheuklappen und Voreingenommenheit."

\subsection{Biographie Kemal (m, Herkunftsstaat Türkei)}

Kemal ist 52 Jahre alt. Er stammt von der Schwarzmeerküste der Türkei. Nach seinem Abitur in Istanbul kam er 1978 nach Deutschland. Der Vater war Schuster. Kemal hat drei Brüder und drei Schwestern. Zwei seiner Geschwister haben nicht studiert. Er stand nach dem Abitur vor der Entscheidung, in der Türkei zu bleiben und bei den damaligen politischen Unruhen sich einen Weg zu suchen 
oder auszuwandern. „Der Weg, zu bleiben, wäre sicherlich definitiv nicht der bessere Weg gewesen," sagt er.

Unter dem Einfluss seines älteren Bruders zog er zu diesem nach Deutschland, um dort zu studieren. Der Bruder war schon längere Jahre in Deutschland, hatte studiert und war als Lehrer tätig. Kemal besuchte einen Sprachkurs und anschließend ein Studienkolleg, damit er in Deutschland ein Studium aufnehmen konnte. Er arbeitete während seines Studiums nebenbei als Kellner. Aus finanziellen Gründen musste er sein Studium unterbrechen. Später schloss er das Studium der Elektrotechnik als Diplom-Ingenieur ab. Er arbeitete zuerst bei einem Planungsbüro und wickelte verschiedene Projekte im Auftrag eines großen Chemiekonzerns ab. Später war er als Gruppenleiter und Projektverantwortlicher tätig und für die Akquisition von Neuaufträgen zuständig. Mitte der 1990er Jahre wechselte er zu einem Automobilhersteller. Er bildete sich weiter fort, absolvierte ein Aufbaustudium als Planer und schloss während seiner Tätigkeit ein weiteres Studium als Wirtschaftsingenieur ab. Er stieg bis zum Abteilungsleiter im Entwicklungsbereich auf und trägt heute die Verantwortung für über 100 Mitarbeiter. Er sagt: „Bis ich es hierher schaffen konnte, musste ich viele Hürden überwinden. Es findet Mobbing auf allen Ebenen statt. Sogar Vorgesetzte mobben. Einer sagte mir mal, als Türke hast Du den ,Schwarzen Peter' in der Hand. Das gab mir zu denken. Ich musste immer wieder erfahren, wenn man erfolgreich ist, wird dein Erfolg mit der Kehrseite des Feldstechers angeschaut, aber deine Fehler werden mit der Lupe betrachtet. Das waren manchmal anstrengende Zeiten. Als Türkischstämmiger ist deine Karriereleiter nach oben gedeckelt. Das Betriebsklima ist dennoch gut, Einzelfälle von Diskriminierung nehme ich nicht ernst. Diese können nicht für alle stehen. Unser Unternehmen versucht Diversity Management zu leben und umzusetzen."

Über sein Privatleben erzählt er, dass er seine Frau in einem Verein, in dem er sich ehrenamtlich engagierte, kennenlernte. Sie hat Sozialpädagogik studiert. Sie heirateten und bekamen zwei Söhne. Das soziale Umfeld besteht sowohl aus deutschen als auch nichtdeutschen Personen. Freundschaften werden seit den Studienzeiten gepflegt und weitergeführt.

Die Nachbarschaft ist überwiegend deutsch. „Unsere Kinder lernen beide Kulturen. Ihre Freunde sind überwiegend Deutsche. Wir legen sehr viel Wert auf 
Sprache, insbesondere auf die Muttersprache. Denn wer seine Sprache gut spricht, lernt auch weitere Sprachen leichter. Meine Frau und ich achten immer darauf, dass die deutsche Sprache und der Schulerfolg streng gefördert werden. Wenn mein jüngerer Sohn drauf angesprochen wird, sagt er, er sei ein Deutscher. Ich empfinde dieses nicht als schlimm. Das Kind muss seine Identität entwickeln. Diese Identität wird selbstverständlich von der deutschen Gesellschaft auch geprägt sein. Wir bekennen uns zu diesem Land, wollen aber auch, dass dieses Land uns aufnimmt. Wir wollen in diesem Land friedlich und mit Pflichten, aber auch mit Rechten zusammenleben."

Abschließend stellt Kemal seine Position zur Integration unmissverständlich dar. Er sagt, dass die Integration in Deutschland erforderlich ist, sonst würde man nie in der Lage sein, seine eigenen Vorstellungen zu leben oder sich anzupassen. Jedoch müssten auch die gesetzlichen Rahmenbedingungen und die Strukturen für die Förderung der Integration geschaffen werden. „Wir sind bereit, unseren Beitrag dazu zu leisten. Das machen wir im kleinen Rahmen bereits jetzt. Wir sorgen dafür, dass unsere Kinder höhere Bildungsabschlüsse anstreben.“ Sein abschließender Satz ist für Kemal die Definition von Integration: „Der Erfolg der Integration hängt entscheidend von der Bildungsförderung und dem späteren Bildungsniveau der jungen Migranten ab. Eine bessere und sinnvollere Investition als in die Bildung sehe ich nicht.“

\section{Fazit und Ausblick}

Der Begrifflichkeit von Zugehörigkeit mögen komplexe Verständnisse zugrunde liegen. Aus diesem Grund wird an dieser Stelle der Begriff und die Konzeption von Integration aufgegriffen, die auch die Interviewten verwenden, da deren verschiedene Definitionen, Konzepte und Auslegungen hinreichend vorgenommen und diskutiert wurden. ${ }^{32}$

Von der etymologischen Bedeutung des Begriffs ausgehend bedeutet Integration Einbeziehung oder Ganzheit, je nachdem in welchem Zusammenhang er steht. So geht es um die Schaffung bzw. die Wahrung einer Einheit, die aus verschiedenen Fragmenten zusammengesetzt ist oder die Zusammenführung

\footnotetext{
${ }^{32}$ Vgl. u. a. im soziologischen Kontext Hartmut Esser 2009; im Kontext Bildung Rolf Becker 2011.
} 
verschiedener Teile in ein Ganzes. ${ }^{33}$ Im sozialpolitischen Kontext meint „Integration den Prozess, durch den bisher außen stehende Personen oder Gruppen zu Gliedern oder Teilen einer größeren sozialen Gruppe oder einer Gemeinschaft werden “. ${ }^{34}$ Dieser verschiedene Dimensionen (kognitive, strukturelle, gesellschaftliche und emotionale) umfassende Prozess wird sowohl durch die Orientierungen der Zuwanderer als auch durch gesellschaftliche und politische Rahmenbedingungen beeinflusst und kann unterschiedliche Verläufe nehmen: Assimilation als Übernahme der Aufnahmekultur bei Aufgabe der Herkunftskultur, Segregation als Beibehaltung der Herkunftskultur ohne Annäherung an die Aufnahmekultur, Inklusion als Einbindung in die Gesellschaft ohne Übernahme der Aufnahmekultur und Exklusion als Ausschluss aus der Gesellschaft trotz Übernahme der Herkunftskultur. ${ }^{35}$

Wir vertreten das Verständnis, dass es sich bei Integration nicht um eine vollständige Anpassung an eine bestehende Gruppe oder Gesellschaft handeln soll. Vielmehr folgt aus der Integration der aufzunehmenden Gruppe(n) eine Neukonstruktion und ein damit einhergehend verändertes Ganzes, wodurch ein soziokultureller Wandel angestoßen wird. ${ }^{36}$ Integration im sozialen Sinn bedeutet stets auch eine „aktive Teilhabe am und die subjektive Identifikation mit dem größeren Ganzen“. ${ }^{37}$

Integration ist ein langfristiger Prozess. Sein Ziel ist es, alle Menschen, die dauerhaft und rechtmäßig in Deutschland leben, in die Gesellschaft einzubeziehen. Zuwanderern soll eine umfassende und gleichberechtigte Teilhabe in allen gesellschaftlichen Bereichen ermöglicht werden. Sie stehen dafür in der Pflicht, Deutsch zu lernen sowie die Verfassung und die Gesetze zu kennen, zu respektieren und zu befolgen. ${ }^{38}$

Integration basiert mithin auf Wechselseitigkeit und damit vor allem auf der beidseitigen Bereitschaft, die damit verbundenen Herausforderungen anzunehmen und zu gestalten. Außerdem gilt als unbestritten, dass Offenheit und Toleranz Basis für Integration sind, allerdings müssen diese Grundpfeiler und die

\footnotetext{
${ }^{33}$ Vgl. Wolfgang Vortkamp 2008, S. 65.

${ }^{34}$ Vgl. ebd.

${ }^{35} \mathrm{Vgl}$. Hartmut Esser 2009.

${ }^{36}$ Vgl. Wolfgang Vortkamp 2008, S. 65.

${ }^{37}$ Ebd.

${ }^{38}$ BMI 2011.
} 
mit ihnen verbundenen Maximen und Regeln stets wechselseitige Beachtung finden. Wir verstehen sie als umfassende gesellschaftliche Teilhabe, die unter anderem die Bereiche Bildung und Arbeit umfasst und dabei auch den Aspekt der Identifikation einschließt. Spätestens hierdurch gilt ein besonderer Blick den Muslimen, weil sich ein Teil ihrer Identitätsbildung über ihren Glauben, den Islam, vollzieht.

Insgesamt kann festgehalten werden: Migranten in Deutschland, auch Muslime, haben sich zunehmend stärker eingefügt oder sind auf einem guten Wege dorthin, auch wenn „ein großer Teil sehr viel traditioneller als die Deutschen eingestellt ist und nach wie vor zu seinen kulturellen und religiösen Wurzeln steht. Insbesondere betrifft dies die türkischstämmigen Migranten “. ${ }^{39}$ Einen besonderen Einfluss auf den Wertekanon Deutschlands hat die Migration der vergangenen Jahrzehnte also nicht nach sich gezogen und wird dies auch nicht. Insbesondere, da die Werteunterschiede sich auf eine religiös-konservative Weltanschauung (von Muslimen) reduzieren, die zudem in Deutschland selber noch bis in die 1970er in ähnlicher Weise ausgeprägt war. Dieser Konservatismus hängt eng mit den Personen, die nach Deutschland migriert sind, zusammen, weil ein Faktor ihrer Identitätsbildung hier in Deutschland das Bewahren von Traditionen ist.

Es gilt dennoch festzuhalten, dass der Wertekanon und damit das gesellschaftliche Zusammenleben durch die Migranten nicht gefährdet ist.

Dennoch sollten noch größere Anstrengungen unternommen werden, um den Prozess des „heimisch Werdens" der Muslime in Deutschland und Europa zu begleiten, und umgekehrt sind die Muslime Europas vor die Herausforderung gestellt, ihr Glaubensverständnis zu modernisieren. ${ }^{40}$

Demografie und wirtschaftliche Standortfaktoren bestimmen maßgeblich die Attraktivität eines Landes. Die Konzentrationsprozesse bei der Verdichtung von wirtschaftlicher Aktivität in einer bestimmten Region werden durch die Heterogenität von Arbeitskräften vor Ort beschleunigt. Somit kann es einen Vorteil darstellen, wenn Unternehmen bei der Auswahl ihrer Arbeitskräfte aus der

\footnotetext{
${ }^{39}$ Unabhängiges Meinungsforschungsinstitut INFO und LILJEBERG Research International 2010, S. 12 .

${ }^{40}$ Wolfgang Schäuble 2008, S. 8.
} 
Standortbevölkerung auf einen großen Pool von (jüngeren!) Menschen mit Migrationshintergrund zurückgreifen können. Steinrücken und Jaenichen stellen in einer ihrer Arbeiten zur Wirtschaftsleistung von Ausländern die These auf, dass diese Fähigkeiten, Fertigkeiten und Kenntnisse besitzen, die kombiniert mit dem Humankapital der Mehrheitsgesellschaft zu höherem Pro-Kopf-Einkommen des jeweiligen Standorts führen können. ${ }^{41}$ Denn Zuwanderung führt zu einer Verdichtung von „Humankapital“ in bestimmten Regionen, wodurch sich die Ansammlung verschiedener Unternehmen an einem Standort erhöht und so die betroffenen Regionen eine überdurchschnittliche wirtschaftliche Entwicklung erfahren können.

Das Zulassen verschiedener Denkstile, Religionen und kulturellen Unterschiede kann zu einem interkulturellen upgrade in der Gesellschaft führen, wodurch die Möglichkeit einer wechselseitigen Anhebung der Fähigkeiten und Fertigkeiten durch das gemeinsame Leben der verschiedenen Gruppen besteht. Sobald ein solcher Lernprozess zwischen den Gruppen stattfindet, verschwinden die Unterschiede in den aufgrund unterschiedlicher Sozialisation festgelegten Fähigkeitsbündeln mit der Zeit.

Wenn sich die Fähigkeitsbündel der Gruppen angeglichen haben, ist die Gesamtbevölkerung im Hinblick auf die entsprechenden Eigenschaften für den betrachteten Standort wieder homogen und muss durch weitere Zuwanderung neue Impulse erfahren. Für eine lenkende Einwanderungspolitik bedeutet dies, dass das Qualifikationsniveau der Zuwanderer in bestimmten Bereichen nicht zwingend über dem der nativen Bevölkerung liegen muss, sofern es sich bei den mitgebrachten Qualifikationen der Migranten um andere als die der Mehrheitsbevölkerung handelt. Dies gilt selbst für formal weniger gebildete Migranten, die ebenfalls den Prozess bereichern und erst recht für Migranten mit solchen Biografien, wie sie in diesem Beitrag wiedergebenen worden sind.

\section{Literatur}

Becker, Rolf, Integration von Migranten durch Bildung und Ausbildung-theoretische Erklärungen und empirische Befunde, in: ders. (Hrsg.): Integration durch Bildung, Wiesbaden 2011 , S. 11-36.

\footnotetext{
${ }^{41}$ Vgl. Torsten Steinrücken und Sebastian Jaenichen 2004.
} 
Berlin-Institut für Bevölkerung und Entwicklung (Hrsg.), Ungenutzte Potentiale. Zur Lage der Integration in Deutschland, Berlin 2009.

Bertelsmann Stiftung (Hrsg.), Zuwanderer in Deutschland. Untersuchung des Instituts für Demoskopie Allensbach, Gütersloh 2009.

BMI - Bundesministerium des Innern, Migration und Integration. Aufenthaltsrecht, Migrations- und Integrationspolitik in Deutschland, Berlin 2011.

Esser, Hartmut, Pluralisierung oder Assimilation? Effekte der multiplen Inklusion auf die Integration von Migranten, in: Zeitschrift für Soziologie, Jg. 38, Heft 5, Wiesbaden 2009, S. 358-378.

Institut für Mittelstandsforschung (Ifm) der Universität Mannheim (Hrsg.), Kurzfassung der Studie: Die Bedeutung der ethnischen Ökonomie in Deutschland, Mannheim 2005.

Kober, Ulrich, Zugehörigkeit als Schlüssel zur Integration, S. 11-16. In: Wer gehört dazu?, Bertelsmann Stiftung, Gütersloh 2011.

Schäuble, Wolfgang am 22.11.2008: Religiöse Vielfalt und gesellschaftlicher Zusammenhalt in Deutschland, unter: http://www.bmi.bund.de/SharedDocs/Reden/DE/2008/11/ bm_eugenbieserpreis.html?nn = 268184 (10.10.2012).

Sinus Sociovision, Zentrale Ergebnisse der Sinus-Studie über Migranten-Milieus in Deutschland unter: http://www.sinus-institut.de/uploads/tx_mpdownloadcenter/ MigrantenMilieus_Zentrale_Ergebnisse_09122008.pdf (10.10.2012).

Statistisches Bundesamt, Bevölkerung und Erwerbstätigkeit, Bevölkerung mit Migrationshintergrund, - Ergebnisse des Mikrozensus 2010 - Fachserie 1, Reihe 2.2, Wiesbaden 2011/1.

Statistisches Bundesamt, Statistisches Jahrbuch 2011 für die Bundesrepublik Deutschland mit Internationalen Übersichten, Wiesbaden 2011/2.

Statistisches Bundesamt, Bildung und Kultur, Allgemeinbildende Schulen, Fachserie 11, Reihe 1, Wiesbaden 2011/3.

Statistisches Bundesamt, Bevölkerung und Erwerbstätigkeit, Ausländische Bevölkerung Ergebnisse des Ausländerzentralregisters, Fachserie 1, Reihe 2, Wiesbaden 2012/1.

Statistisches Bundesamt, Bevölkerung und Erwerbstätigkeit, Haushalte und Familien, Ergebnisse des Mikrozensus, Fachserie 1, Reihe 3, Statistisches Bundesamt, Wiesbaden $2012 / 2$.

Statistisches Bundesamt, Wirtschaft und Statistik, Statistisches Bundesamt, Wiesbaden September 2012/3.

Statistisches Bundesamt, Bevölkerung und Erwerbstätigkeit, Wanderungen - Fachserie 1, Reihe 1.2, Wiesbaden 2012/4.

Statistisches Bundesamt, Fachserie 1, Reihe 2.2 (Ergebnisse des Mikrozensus 2011), Wiesbaden 2012/5.

Statistisches Bundesamt, Fachserie 2, Reihe 1, Einbürgerungen, Wiesbaden 2012/6. 
Steinrücken, Torsten und Jaenichen, Sebastian, Zum Einfluss von Ausländern auf die Wirtschaftsleistung von Standorten - Ist Zuwanderung ein Weg aus der ostdeutschen Lethargie?, Oktober 2004. (Diskussionspapier Nr. 41).

Unabhängiges Meinungsforschungsinstitut INFO und LILJEBERG Research International, Wertewelten von Deutschen und Migrant/innen - Migration zwischen Integration und Ausgrenzung, Berlin und Ankara 2010.

Vortkamp, Wolfgang, Integration durch Teilhabe. Das zivilgesellschaftliche Potenzial von Vereinen, Frankfurt/M. 2008.

Niemals zuvor haben so viele Muslime dauerhaft in Deutschland gelebt. Aber sind sie hier auch zu Hause? Fühlen sie sich wohl, gehören sie zu „uns“, ${ }^{42}$ und gehört ihre Religion, der Islam, zu Deutschland? Diese Fragen wurden lange Zeit durch politische Rabulistik ersetzt, ob Deutschland nun Zuwanderungsland sei oder nicht. Durch diese realitätsferne Debatte wurden entscheidende integrationspolitische Weichen nicht gestellt, Menschen weiterhin ausgegrenzt - und Zeit vertan.

Teile der Politik hinken gesellschaftlichen Entwicklungen auch in diesem Politikfeld hinterher: Inzwischen verbindet allerdings ein Teil der Menschen der Mehrheitsgesellschaft mit der Zuwanderung von Muslimen nach Deutschland und Europa die Annahme, dass diese nunmehr auch zu Deutschland (und Europa) gehören. Die kleine, aber feine Unterscheidung zwischen den Muslimen und ihrer mitgebrachten Religion, dem Islam, macht das Gros dabei allerdings nicht.

Bestandteil ihrer Annahme ist vielmehr, dass Muslime wie Nichtmuslime über so starke kulturelle, gesellschaftliche, religiöse und sonstige Gemeinsamkeiten verfügen, dass die Unterschiede überdeckt werden. Diese Menschen verstehen die Attraktivität Deutschlands als westliche, säkulare, rechtstaatliche und kriegsmüde Gesellschaft mit „freier“ Lebensweise, der auch Muslime auf längere Sicht nicht widerstehen könnten und die sie ihrer eigenen Tradition vorziehen würden. Nicht unbedingt wird damit auch der Gedanke verbunden, dass Muslime ihre religiös-kulturellen Wurzeln aufgeben und gewissermaßen als unauffällige Gläubige in die Gesellschaft eintauchen sollen.

\footnotetext{
${ }^{42}$ Bei der regelmäßigen Erhebung des „Integrationsbarometers“ beauftragte die NRW-Stiftung mit Unterstützung der Mercator-Stiftung den Sachverständigenrat deutscher Stiftungen für Integration und Migration, der bei 93,5\% der Migranten in NRW ein „Heimatgefühl“ ermittelte, vgl. Die NRWStiftung, Düsseldorf 2/2011, S. 11/12.
} 
Gleichwohl reden gerade Verfechter eines Multikulturalismus und solche, die sich im ,interreligiösen Dialog“ engagieren, das Wort von gemeinsamen kulturhistorischen Strängen der „abrahamitischen Religionen“ und der Zeit, die irgendwann alle Unterschiede in respektvollem Umgang, in Toleranz und Miteinander auflösen sowie Angst vor Überfremdung und Verlust von tradierten Werten marginalisieren würde. Beide Positionen sind vermeintliche Schlüsselwörter im Zusammenleben von Menschen unterschiedlicher Ethnien und Religionen, aber bei beiden ist keine gesellschaftspolitische Strategie erkennbar. Dafür stellen sie das Rechts-Links-Schema infrage, nähren allerdings auch die Fiktion von Integration, die sich von selbst einstellt, wenn ihr nur Zeit gegeben und miteinander gesprochen wird. Dennoch sind beide Ansätze heilsame Stachel für ein Zuwanderungsland, weil sie an die Pluralisierung der sozialen Lebenswelten erinnern, Gemeinsames statt Trennendes und nicht soziale Semantiken und symbolische Grenzen von Teilen der Gesellschaft betonen.

Auch gelegentliche Fraternisierungen deutscher Kirchenvertreter mit ihren Kollegen in den islamischen Dachverbänden zu deren religionspolitischen Forderungen sind nicht wirkliche Annäherungen, dienen vielmehr eigenen Interessen, in Deutschland keine Diskussion über das tradierte Staatskirchenrecht aufkommen zu lassen, dem die Kirchen manches Privileg verdanken.

Die auf solchen Versuchen gründenden Integrationshoffnungen überdeckten lange Zeit die eigentliche Frage, die der Leitidee einer „deutschen Perspektive" des Islam. Anders ausgedrückt: Sind Säkularisierung, Pluralismus, Demokratie und Rechtstaatlichkeit überhaupt mit einer islamischen Identität vereinbar? Kann es einen Islam geben, der in den Werten des aufklärten Europa seine eigenen erkennt und realisiert? Hat eine solche Leitidee in einer Zeit eine Chance, verwirklicht zu werden, in der der politische Islam als Folge der weitgehenden politischen, gesellschaftlichen und wirtschaftlichen Perspektivlosigkeit islamisch geprägter Staaten sowie der Wertekrise, in der Europa steckt, erstarkt und sich manche Muslime dabei gerade die Traditionen ihrer Religion als Anker suchen?

Für Ednan Aslan, Professor für islamische Religionspädagogik an der Universität Wien, liegt die Lösung in einer theologischen Neubesinnung des Islam:

Die islamische Theologie hatte bisher viele Regeln, die unserem Menschenrechtsverständnis widersprechen, wie die ungleiche Behandlung von Juden und Christen oder 
die Steinigung. Diese Regeln sind nicht absolut. Wir sind in der Lage, eine neue Theologie zu definieren. ${ }^{43}$

Mit einer solchen Vorstellung verbunden ist zwangsläufig die gemeinsame Verständigung islamischer Gruppierungen in Deutschland darauf, demokratische und rechtstaatliche Prinzipien sowie die Trennung von Staat und Religion anzuerkennen, die Menschenrechte uneingeschränkt zu respektieren und ihre Erfahrungen in Solidarität und Toleranz mit denen anderer Gemeinschaften zu vereinen.

Das sind hohe, aber unverzichtbare Anforderungen, für deren Einlösung allerdings auch - sofern sie die uneingeschränkte Akzeptanz der Grundwerte des Grundgesetzes übersteigt und sich auf theologische Korrekturen bezieht - Zeit eingeräumt werden muss.

Die Autorinnen und Autoren haben in ihren Beiträgen viele Facetten des „Zugehörigkeitsprojekts Deutschland“ aufgezeigt, zahlreiche Fragen beantwortet und viele neue generiert. Dabei wurden Sollbruchstellen freigelegt, die auf „islamischer Seite“ sichtbar werden und Zugehörigkeit ausschließen können, zumindest erschweren: Ist der Islam, den die Dachverbände in Deutschland repräsentieren, derjenige, den Aslan neuerdings meint? Warum wird eine kritische Auseinandersetzung mit extremistischen Auslegungen islamischer Glaubenslehren als zentrales Thema islamischer Gemeinschaften und Organisationen nicht öffentlich geführt? Was tun die Dachverbände, damit ihr Selbstverständnis als religiöse Minderheit und als Bürgerinnen und Bürger in einem demokratischen Verfassungsstaat nicht in Frage gestellt werden kann? In welchem Verhältnis stehen Mainstreamislam und Islamisierung? Wie können Gesellschaft und islamische Repräsentanz der Problematik von Segregation und der sukzessiven ideologischen Radikalisierung innerhalb islamistischer Netzwerke begegnen? Ist die Scharia, das islamische Rechtssystem, für den Islam in allen Tei-

\footnotetext{
${ }^{43}$ So auf einer Rotary-Veranstaltung und zitiert in der Wiener Zeitung vom 27.09.2008, unter: http://islamische-religionspaedagogik.univie.ac.at/fileadmin/user_upload/proj_muslime_eu/presse/Europ\%C3\%A4isch er_Islam_.pdf (30.09.2012). Bis dahin hatte sich Aslan nur mit konservativ-islamischen Positionen zu Wort gemeldet. Diese scheint er - jedenfalls in dem zitierten Vortrag - zugunsten eines gesellschaftlich tragfähigen Ansatzes aufgegeben zu haben, vgl. dazu seine bisherigen Positionen und die Kritik daran von Assia Maria Harwazinski, Islami(sti)sche Erziehungskonzeptionen: Drei Fallbeispiele aus Baden-Württemberg, Marburg 2005.
} 
len substanziell, oder ist ein Islam vorstellbar, der ohne diejenigen Teile auskommt, die gegen Menschenrechte und Menschenwürde verstoßen? Und wenn es - hier bereits der Kompromiss - lediglich um eine zeitgemäße Interpretation der Scharia geht: Kann in Deutschland, in Europa, von der dominierenden Praxis abgewichen werden, nur auf die Rechtstraditionen der ersten Jahrhunderte zurückzugreifen, oder wird „das Tor des eigenständigen Bemühens“, der Idschtihad, wieder geöffnet? Wird mit der Implementation der islamischen Theologie und Religionspädagogik an deutschen Universitäten und Schulen zugleich eine islamische Lehre grundgelegt, die notwendige Erneuerungen unter Wahrung substanzieller Tradition vorantreibt?

Für dieses Konvolut an Fragen können Muslime - wenn sie denn als Integration fördernde Perspektiven im Interesse eines „Zugehörigkeitsprojekts Deutschland" in Angriff genommen werden - erwarten, dass ihre Wurzeln, Kultur und Religion respektiert, sie in den weiteren Ausbau der Zivilgesellschaft einbezogen, uneingeschränkt an der Entwicklung aller gesellschaftlichen Bereiche beteiligt werden und Zugang auf dem Arbeitsmarkt, zu öffentlichen Ämtern u. a. m. entsprechend ihrer Qualifikationen erhalten.

Dieser Weg ist lang und stellt auch - wenige, auf dem Grundgesetz basierende - Bedingungen als Vorleistung an Muslime. Wie steinig er ist, zeigen zudem Versäumnisse, absichtsvolle Diskriminierungen, unverhohlener Rassismus sowie institutionelle Erschwernisse in Alltags- und Rechtsbereichen gegenüber Muslimen. Sie zeigen sich täglich, kommen bislang offensichtlich widerstandslos aus der Mitte der Gesellschaft und benötigten nicht erst Sarrazin als Sprachrohr: Was versteht die Mehrheitsgesellschaft darunter, wenn sie von „ihren“ Werten, „ihrer“ Kultur und „ihren“ Traditionen spricht? Kann sie sich dabei auf gemeinsame Nenner einigen, oder sind es Phrasen, die lediglich für eine Abwehrrhetorik herhalten müssen? Wird ein Kulturbegriff verwendet, der „westliche“ Kultur der islamischen als überlegen ansieht? Akzeptiert die Gesellschaft auch dann eine Unteilbarkeit des Rechts, wenn es um ,islamische“ Fragen wie Kopftuch, Gebetsräume, nach Geschlechtern getrennten Unterricht oder aktuell - um islamischen Religionsunterricht oder Beschneidungen geht? ${ }^{44}$ Wie

\footnotetext{
${ }^{44}$ In der im Sommer 2012 in den Medien geführten Diskussion über Beschneidungen von Jungen wurden erst Argumente für Beschneidungen ausgetauscht und erwogen, als Juden in dieser Frage mit Muslimen einen argumentativen Schulterschluss herstellten.
} 
halten es Schulen und Betriebe mit der Bildungs- und Berufsinklusion jenseits von Rasse, Ethnie, Geschlecht oder Religion? Warum findet eine Verengung der Berichterstattungen über den Islam auf Themen wie Terror, Fundamentalismus, Gewalt, Bedrohung und Rückständigkeit statt? Warum wird als Folge dieser massenmedial verbreiteten Szenarien eine islamistische Unterwanderung der Gesellschaft in Deutschland suggeriert und damit zugleich die Möglichkeit des rationalen Diskurses geschwächt? Inwieweit wirkt das Wechselverhältnis, das Stereotypen als kognitive Dimensionen von sozialer Diskriminierung darstellt, auf die gesamte Gesellschaft zurück? Inwieweit entsprechen Diskriminierte den Bildern, die von ihnen in groben Strichen gemalt werden, und inwieweit wird bedacht, dass Viktimisierung und Selbstviktimisierung sowie Fremdheitserfahrungen und Segregation jeweils Geschwisterpaare bilden?

Damit sind weitere Hürden skizziert, die bei der Zugehörigkeit des Islam zu Deutschland, nunmehr von "nichtislamischer" Seite aufgebaut, aber eher heute als morgen wieder eingerissen und durch einen Dialog ersetzt werden müssen. Denn es ist zunehmend schwierig, sich eine junge Generation in Deutschland vorzustellen, in der sich „die“ Muslime und „die“ Mehrheitsgesellschaft gegenseitig als Kollektive gegenüberstehen, von denen gegenseitige Anpassungsleistungen gefordert werden. Notwendig erscheint vielmehr gesellschaftliche Kohäsion, insbesondere nach bestehenden und zu vermittelnden Gemeinsamkeiten verbindlicher Wertvorstellungen, die die Gesellschaft zusammenhält. Dabei müssen Haltungen überdacht werden, denn wer sich als der oder die „Andere“ denkt, kann sich kaum auf eine Perspektive von Gemeinsamkeiten, Ähnlichkeiten oder Zugehörigkeit einlassen. Erwartet werden darf von Muslimen wie Nichtmuslimen, die Gratwanderung der Bestimmung des „Eigenen“ und „Anderen“ im Sinne gemeinsamer gesellschaftlicher Vorstellungen zu reflektieren und sie nicht bei Bedarf wechselseitig strategisch einzusetzen, um pauschale Zumutungen und Vorwürfe gegenüber der Gruppe zu formulieren und abzuwehren oder Loyalitäten von Gruppenmitgliedern einzufordern. Alle weiteren Dimensionen von Identität und eben auch andere soziale Bindungen der Einzelnen würden sonst leicht aus dem Blick geraten. Erwartet werden kann auch, zu einem Sprachgebrauch zurückzukehren, der vor 9/11 gang und gäbe war, nämlich bei Notwendigkeit Menschen nach ihrem Namen, ggf. auch nach ihrer 
Nationalität, nicht aber, wie bei Muslimen heute üblich, nach ihrer Religion zu bezeichnen.

$\mathrm{Zu}$ wünschen ist eine Politik, die nicht nur Integrationsprobleme sieht und sie in Fachzirkeln bespricht, sondern sie im öffentlichen Diskurs mit der Gesellschaft zügig löst, ohne bestehendes Recht zu dehnen oder auf Ausnahme-, Übergangs- und Befristungsregelungen zu setzen. Alle Menschen in Deutschland, ob die der Mehrheitsgesellschaft oder Migranten, Muslime oder Angehörige sonstiger Religionen und Ethnien, haben Anspruch auf nachhaltige Ergebnisse.

Dieser Diskurs muss zudem ein Integrationsklima stärken, in dem Muslime Subjekte, nicht Objekte deutscher (oder türkischer!) Politiker, Verbandsvertreter oder von Medien sind, sondern Zugehörigkeitsgefühle zu Deutschland entwickeln können, die erwidert werden. Muslime wie Nichtmuslime müssen darin gestärkt werden, sich gegen Vorurteile sowie Pauschalierungen auch und gerade dann zu immunisieren, wenn sie durch religiöse Beleidigungen samt massiven Gewaltreaktionen - die sich irgendwo in der Welt ereignen und die politisch instrumentalisiert werden - auch in Deutschland gegeneinander in Stellung gebracht werden. Dabei müssen sie gemeinsam lernen, dass es konkurrierende Verfassungsnormen gibt, zu denen neben der Religions- auch die Presse- und Meinungsfreiheit gehören, deren Verhältnis zueinander kooperativ und gewaltlos immer wieder neu und fallorientiert bestimmt werden muss:

Verfassungsrechtlich geschützte Rechtsgüter müssen in der Problemlösung einander so zugeordnet werden, dass jedes von ihnen Wirklichkeit gewinnt. Wo Kollisionen entstehen, darf nicht in vorschneller „Güterabwägung“ oder gar abstrakter „Werteabwägung" eines auf Kosten des anderen realisiert werden. Vielmehr stellt das Prinzip der Einheit der Verfassung die Aufgabe einer Optimierung: beiden Gütern müssen Grenzen gezogen werden, damit beide zu optimaler Wirksamkeit gelangen können. ${ }^{45}$

Die für die Verfassungsrechtsprechung der Gegenwart vielleicht wichtigste Einsicht liegt darin, dass bei kollidierenden Verfassungswerten nicht nach dem Vorrang zu fragen ist, sondern nach einem Ausgleich im Sinne praktischer Konkordanz, bei dem die „Würdeidee“ von Art. 1 Abs. 1 GG die Erkenntnis leitet. Auch wenn dieser Begriff unscharf erscheint, ermöglicht er gerade deshalb,

\footnotetext{
${ }^{45}$ Konrad Hesse, Grundzüge des Verfassungsrechts der Bundesrepublik Deutschland, Neudruck 20. Auflage, Heidelberg 1999, S. 28, Rn 72.
} 
Rechtskonflikte gesellschaftlichen Herausforderungen entsprechend der Grundwerte des Grundgesetzes anzupassen. ${ }^{46}$

Damit sind zugleich die zentralen Ziele einer Erziehung beschrieben, die die Bildung eines reflektierenden kritischen Individuums und seiner Handlungskompetenzen anstrebt und damit vermeidet, identitätspolitische Positionen eines Kollektivs von Muslimen oder Nichtmuslimen auszubilden.

${ }^{46} \mathrm{Ob}$ dieser Gedanke letztendlich auch bei der Lösung der „Beschneidungsfrage“ berücksichtigt wird, wird Ende 2012/Anfang 2013 im Gesetzgebungsverfahren des Bundestages entschieden. 
